\title{
3 LA UNIVERSIDAd PÚBlica Y EL PODER POLÍ́tico EN ARgENTINa dURANTE EL SIGLO XX
}

\author{
Lic. Mabel Inés Falcón
}

Dr. Angel Rodríguez Kauth

Facultad de Ciencias Humanas

Universidad Nacional de San Luis. Argentina

\section{INTRODUCCIÓN}

Las relaciones de la Universidad -como institución enseñante-con los poderes políticos nacionales, siempre han sido difíciles y hasta complicadas. Esto se ha debido a que la Universidad es un apetecible «bocado" dónde echar pie a tierra como territorio de conquista para las huestes que han triunfado en una campaña electoral.

Históricamente, en Argentina, esto se dio hasta 1918 con la aparición del episodio de la Reforma Universitaria, que tuvo entre sus líderes a personajes insignes de la historia nacional, como lo fueron D. Roca, J. Ingenieros, A. Palacios y J. V. González, entre muchos otros de aquella época productora de pensadores libres -o librepensadores- como les gustaba llamarse así mismos. Hasta la publicación del Manifiesto Liminar Reformista, que vio la luz en la Ciudad de Córdoba y cuyas consecuencias, la Reforma Universitaria, se extendió como un reguero de pólvora por todo el país y buena parte de Latinoamérica, las consecuencias del éxito político de la Reforma Universitaria, se manifestaron en primer término en la designación de profesores. Hasta ese momento, los profesores eran seleccionados y ubicados en una terna a través de un concurso con un jurado académico, pero era el Presidente de la República el que se reservaba el derecho de designar dentro de la terna, a 
quien él deseaba. Prueba de esto, aún se puede encontrar en las páginas inmortales que dejó impresas José Ingenieros en una de sus obras más populares: "El Hombre Mediocre» (1913); de las mismas puede recogerse la manera cómo eran designados los profesores en la Universidad Argentina, entre otras de las múltiples características perversas de una relación insana entre el Poder Ejecutivo -de fuerte raigambre oligárquica para ése entoncesy la institución universitaria.

Desde 1918 en adelante, la situación sufrió cambios insospechados en el orden académico en cuanto a la designación de docentes -como la periodicidad de la cátedra- y en la relación docente-alumno. Sin embargo, los embates del Poder político contra las Universidades se han venido sucediendo de manera sistemática, sobre todo en épocas de gobiernos inconstitucionales con base de apoyo en las Fuerzas Armadas de la Nación.

Tal enojosa situación se vivió tanto luego del golpe militar de 1930, que derrocara al Presidente Hipólito Yrigoyen, como bajo la dictadura cívicomilitar de Guido (1962), y en las dictaduras militares, con apoyo cívico, del General J. C. Onganía (1966) y de sus continuadores -todos ellos generalesde ruta hasta 1973. De este período uno de los testimonios por demás elocuente vividos, fue lo que se denominó como «la noche de los bastones largos" ; más tarde, durante el período más obscuro de la historia política argentina, desde 1976 hasta finales de 1983, la Universidad fue objeto de un intenso castigo y persecución, ya que en sus aulas, tanto profesores como alumnos tenían la pésima costumbre de debatir en voz alta y, además, de "pensar feo», según la prístina y esclarecida opinión de aquellos dictadores de turno, encabezados por los genocidas J. R. Videla, E. Massera y su compañía de adláteres cómplices.

Pero no tan sólo durante períodos de dictaduras militares se vivió bajo el signo de la persecución en los claustros universitarios. También ocurrió algo semejante durante el primer gobierno constitucional del General Juan D. Perón -militar él, como se habrá podido observar por su rango-, encontró en los espacios académicos una férrea oposición a su proyecto político, de neto corte populista, tanto en el sector de los alumnos como en el claustro de los docentes. En un primer momento Perón pretendió seducir a los universitarios con prebendas y halagos, pero al advertir que aquéllos no caían en sus redes, optó por la persecución lisa y llana de los mismos. Fue un período en que la Universidad vio pasar a muchos de sus docentes y alumnos por las cárceles del país y, en este momento, recordamos la desaparición física del estudiante Bravo, militante por entonces del Partido Socialista. Sin embargo, Perón no se atrevió a reducir, de manera significativa, los presupuestos con que el Estado Central asiste al financiamiento, sostenimiento, mantenimiento y crecimiento de las Casas de Altos Estudios en el territorio nacional.

Obvio es que cuando asumió el poder político de la Nación la llamada Revolución Libertadora - 1955 hasta principios de 1958- el panorama cambió 
en 180 grados. Volvieron los exiliados de las universidades... y se fueron los docentes amigos del peronismo. Es decir, en la mejor y más fiel política gatopardista, se modificó algo para que todo siguiera igual.

Nuevamente apareció la figura siniestra de los perseguidos políticos, aunque es preciso señalar que durante esa época se le dio un fuerte impulso a la recuperación académica de los institutos de alto nivel de enseñanza e investigación. No puede dejar de recordarse de aquella época la creación del Consejo Nacional de Investigaciones Científicas y Tecnológicas (CONICET) que ha logrado innúmeros éxitos en el campo de la investigación científica y que aún, a la fecha, y en medio de notables peripecias financieras y económicas para mantener sus políticas de investigación, sigue en pie continuando con el desarrollo de proyectos de investigación y de la fundamental "formación de recursos humanos", tan precisa ella para el crecimiento de una "masa crítica" de excelente calidad de investigadores. En este punto es preciso traer a la memoria unas palabras del inolvidable pacifista $M$. Ghandi, cuando dijo que su país, la India, era demasiado pobre como para darse el lujo de no tener investigación básica.

Retomando a la historia política argentina y sus relaciones con las Universidades y siguiendo un criterio de continuidad histórica temporal, es preciso anotar que al gobierno de facto de la Revolución Libertadora le continuó un Presidente constitucional, el Dr. Arturo Frondizi quien, entre otras varias medidas contradictorias con su ideario político, puso en el tapete de la discusión política lo que se conoció como la antinomia "laica o libre». En realidad, tal oposición de contrarios apuntaba a un eje central, cual era la creación de universidades privadas en el país. A la cabeza de tal intentomarchaban los intereses del Episcopado Argentino, que luego vio plasmados sus esfuerzos con la inauguración de la Universidad Católica de Bs. Aires, a la que sucedieron inmediatamente varias corresponsalías en todo el territorio. La llamada Ley Domingorena dio lugar al nacimiento de las universidades privadas con apoyo del Estado nacional o de los Estados provinciales.

Luego del interregno de la autoproclamada "Revolución Argentina» que fuera encabezada por Onganía y que ya citáramos más arriba, se produjo el retorno del peronismo al poder político, hecho ocurrido desde 1973 a 1976. Durante el mismo se produjo un episodio particularmente curioso en la vida política nacional; en ése momento fueron los espacios universitarios uno de los lugares -juntamente con el de los sectores obreros juveniles- que mayor aporte hicieron al retorno de aquél anciano ex Presidente al escenario político nacional. Lo más curioso de este episodio fue la mimetización de amplios sectores intelectuales nacionales, que tradicionalmente se ubicaban en el amplio espectro de la izquierda, para convertirse en fervientes adherentes a la política peronista.

Este extraño hecho de travestismo político e ideológico ocurrió a consecuencia del proceso de autocrítica que hiciera la izquierda argentina luego de 
la expulsión de Perón del poder, en 1955. Episodio este que arrastró a más de un intelectual -como así también a muchos aprendices de tales-a ponerse el rótulo de un emblema partidario que no le correspondía y al cual, incluso, habían combatido (Rodríguez Kauth, 1994).

Esto no fue óbice para que en algunas -la mayoría- de las Universidades Nacionales, comenzara una larga y peligrosa "caza de brujas" respecto a los opositores políticos. Estos no eran solamente los no peronistas, sino también los peronistas de diferente orientación, es decir, de izquierdas o de derechas, aunque es preciso reconocer que fueron los primeros los que sufrieron más cruelmente la persecución y ensañamiento de los organismos paramilitares creados al efecto, entre los cuales no es posible que dejemos de recordar a la tristemente célebre "Triple A» (Alianza Anticomunista Argentina) que fuera comandada por un siniestro personaje como lo fue José López Rega, hombre de confianza de Perón y particularmente de su compañera y más tarde Presidente de los argentinos, Isabel Martínez de Perón.

Parecería que en este tema siempre existe una conjunción adversa en el decurso de los hechos sociales. Un gobierno de signo peronista como el de C. $S$. Menem (1989-1999) que en un principio pareció a dejar a las instituciones universitarias al margen de sus devaneos políticos, luego de un tiempo hizo aprobar en el Parlamento -con mayoría propia- una ley de regulación para las Universidades argentinas que avasalla el principio de la autonomía universitaria, tan caro a los ideales reformistas de 1918. La misma fue recurrida ante la Suprema Corte de Justicia de la Nación por aquellas universidades de mayor tamaño que eran gobernadas por autoridades universitarias no proclives al partido de gobierno, por el contrario, la mayoría de sus autoridades eran -y son- militantes del centenario Partido Radical . Es de hacer notar que el Presidente Menem no se quedó corto ni perezoso a la hora de hacer un desplazamiento de culpas frente a las acusaciones recibidas por parte de los grupos universitarios. A tal fin, vale la pena recordar sus palabras de que "El centro de corrupción en materia de educación está en la Universidad de Bs. Aires» [gobernada por el dirigente radical Oscar Shuberoff]. A lo cual dicho Rector respondió, en la misma publicación, «... que parece que es parte de una operación política de venganza. El Presidente no perdona que la Universidad, junto a otros sectores sociales, haya producido el hecho inédito de forzar al gobierno a dar marcha atrás a una decisión equivocada...».

Las pretensiones del gobierno menemista consiguieron lograr que bajo el saludable y nada desdeñable argumento de la integración de la Universidad con la Comunidad, aquellas se pusieran al servicio de la segunda, en las que están insertas y que las nutre con sus recursos financieros. En realidad, el objetivo menemista era conseguir que las instituciones universitarias comenzaran a introducirse en el proceso de privatización general del país en que estuvo -y está- empeñada la conducción política en el orden nacional. Entre otras medidas poco simpáticas figuraba el proyecto de arancelamiento de los cursos de grado y la explosiva aparición de la oferta de cursos de postgrado, 
como una forma de autofinanciamiento de las universidades, es decir, que generar recursos genuinos desde el punto de vista del análisis financiero, para su mantenimiento, con lo cual el Estado cada vez debería aportar menos dinero para tal objetivo. El sentido final es que las universidades pusieran sus conocimientos y hallazgos al servicio de las empresas privadas vendiendo sus conocimientos a aquellas, aunque los universitarios no supieran muy bien para qué y en qué sentido iban a ser utilizados sus aportes intelectuales de ciencia y tecnología. Este es el punto que con mayor intensidad trataremos en el desarrollo del segundo punto del presente artículo, cuando demos por finalizada con esta pequeña introducción histórica.

El "broche de oro" de esa política que enfrenta a las universidades con el poder político, se produjo durante el primer semestre de 1999, cuando el gobierno nacional, por uno de sus clásicos y remanidos «decretos de necesidad y urgencia" (Rodríguez Kauth, 1998) aplicó un recorte a la educación pública en general de más de 250 millones de dólares, cifra que los presupuestos de la suma total de universidades se traducía en una reducción presupuestaria de casi el $50 \%$. Este episodio fue paradigmático en la historia política del menemismo, ya que, gracias a la movilización popular de los sectores comprometidos con la educación básica y con la educación universitaria en particular, se logró por primera vez en el largo período de este gobierno, que tuviera que poner en reversa la decisión adoptada y, además, produjo la renuncia de la Ministro de Educación de la Nación. Quizás, como expresión de deseos, este episodio haya servido para recuperar la memoria acerca del protagonismo social y político -no solamente de los ámbitos universitarios- sino también en otros sectores de la población tan perversamente castigados por la política económica neoliberal que se ha venido implementando desde su llegada al Poder.

\section{DESARROLLO}

Para llegar a comprender la situación de la Universidad Pública en la realidad política actual de la Argentina, se debe ubicar a la misma en el contexto social y económico que presenta nuestra sociedad. En este caso, vamos a hacer referencia a las condiciones que se manifiestan en la sociedad argentina en el transcurso de la finisecularidad vigesimonónica que estamos transitando.

La crisis en que se ha instalado, a nivel internacional, inclusive dentro de los países de la Europa Occidental que -en su gran mayoría- son gobernados por las socialdemocracias, el llamado Estado de Bienestar, es una crisis que ha venido siendo alimentada desde los sectores hegemónicos del poder $y$, particularmente, desde los poderes económicos y financieros, que vienen pretendiendo terminar con tal expresión de la política estatal para reemplazarla por otra en que el Estado responda solamente a los intereses del capital 
y de los empresarios (Rodríguez Kauth, 1998b). Tal situación de crisis no culmina solamente en una reducción del Estado, o en un corrimiento hacia la oscuridad del mismo de todas aquellas funciones y acciones relacionadas con el crecimiento y salud social de la población.

Las consecuencias más siniestras de la implementación de este modelo económico y social, de claro corte neoliberal económico, han implicado el aumento progresivo y sostenido de una agresiva intervención en la regulación de las diferentes formas de expresarse las relaciones sociales entre los hombres y entre éstos y las instituciones.

Entre las múltiples relaciones sociales citadas se encuentra -para nuestro interés particular en este caso- la institución universitaria que, como todo sistema incluido en otro mayor, se modifica en la medida que las vicisitudes de orden político, y económicas cambian su orientación en función de los mandatos de los sectores de poder. Al respecto, debe señalarse que en Argentina, tales relaciones de poder han sufrido un cambio sustancial en los últimos cincuenta años. Hemos pasado de ser un país conocido con el nombre de "la patria sindical" a ser un una colonia gobernada por «la patria financiera" y el "capital transnacional». Y a estos últimos poco les importa el «bienestar» de los habitantes de cualquier país del mundo; solamente les interesa su rápido enriquecimiento empresario y personal, para lo cual utilizan como testaferros de lujo a agentes nativos que se prestan a tal ejercicio por una buena tajada.

Con respecto a la relación existente entre la universidad y su entorno social, en esta presentación solamente se abordará el aspecto referido a las relaciones existentes y pretendidas -desde las cúpulas del Poder- entre la Universidad y los sectores de la producción asentados en el territorio.

El imaginario social viene siendo -día a día- atravesado por una fuerte tendencia a considerar que para que exista un desarrollo económico y social sostenido, a la vez que para que un país pueda avanzar en su crecimiento, es necesario que se impongan contundentes políticas innovadoras en el campo de lo educativo. Este es el sentido que los organismos internacionales (léase Banco Mundial, Fondo Monetario Internacional, Banco Interamericano de Desarrollo, etc.) le han impreso a las distintas reformas educativas que se están poniendo en práctica en los eufemísticamente conocidos como "países periféricos".

Sin embargo, la condición indispensable para financiar estas reformas educativas consiste en que la oferta que se haga de la misma debe responder en un todo a las demandas formuladas desde el ámbito de la producción, lo cual implica -necesariamente- diseñar estrategias de ajuste entre ambos sistemas, sobre lo cual los argentinos conocemos sobradamente las consecuencias de los «ajustes» económicos y sociales (Rodríguez Kauth, 1994). Desde la perspectiva de los que se benefician con el «ajuste»-que no es precisa- 
mente la gran masa de la población trabajadora y asalariada- la conclusión es simple y sencilla: dónde no hay crecimiento o desarrollo económico y social es debido a que la causa está en el desajuste entre el sistema productivo y el sistema educativo. En consecuencia, para que exista prosperidad aunque fuese como expectativa o fantasía- es preciso que los ajustes respondan a las demandas de quienes lo imponen. Esta ecuación, tan simple, circula no solamente en el discurso de variados matices de políticos de variadas orientaciones ideológicas con mayores o menores posibilidades ciertas de formar o constituir gobierno a la brevedad, sino que también lo hace en el imaginario por el cual transita el hombre común e, incluso, es aceptada y sostenida por muchos científicos de las más diversas disciplinas y, lo que es más llamativo, hasta por quienes se definen como especialistas en educación.

Obvio es que el nivel universitario de enseñanza no puede escapar a esta particular concepción ideológica mercantilista y utilitaria, no obstante, en nuestro país, contrariamente a lo que ocurre en los países centrales -0 , quizás a consecuencia de ello, ya que no somos un país del Primer Mundocomo pretenden hacernos creer las declamaciones sentimentales del Presidente de la República- la universidad y los sectores de la producción nunca han logrado aquella relación idílica que permitiría el supuesto crecimiento económico y el consecuentemente bienestar de la población. En todo caso, hemos tenido un notable desarrollo económico medido a través de indicadores macros, pero la población ha decrecido en su bienestar, a punto tal que luego de la ejecución de tales políticas, más de un tercio de la población vive en lo que hipócritamente se ha definido como "por debajo de la línea de pobreza". Es decir, ya no son ni pobres, se trata de miserables que hacen evocar a la excelente obra del novelista francés Víctor Hugo.

La historia en nuestro país, respecto a las relaciones entre Universidad y producción, comienza cuando los empresarios del sector privado empezaron a constatar la importancia del desarrollo científico y tecnológico en relación a la producción de bienes con valor agregado y, en consecuencia, iniciaron su avalancha de reproches -a las instituciones universitarias estatales- acerca del escaso y pobre aporte que éstas hacían al sostenimiento del aparato productivo de manera indirecta. Está de más decir que en tal circunstancia se olvidó -con aviesa intención- que eran esas mismas instituciones las que producían los recursos humanos que más tarde se instalarían en los espacios productivos, poniendo sus conocimientos al servicio de los mismos. Pero eso era preferible no ponerlo en consideración.

Ante los reclamos que se plantearon por dicha situación y, al no obtener la respuesta esperada por parte de las universidades nacionales, esto es alrededor de la década de 1960, comenzaron a crearse instituciones universitarias privadas a la vez que fundaciones especializadas en algún tipo del conocimiento, muchas de ellas con pretensiones de científicas, que surgieron a la palestra en virtud del fuerte apoyo y aporte empresarial respecto a ambas organizaciones. 
En cuanto hace a la progresión de las universidades privadas, solamente se pueden decir -ante la falta de elementos evaluadores-dos cosas: a) que ellas han facilitado, y continúan facilitando, la mano de obra de las empresas que en más de una oportunidad -como sucede con las organizaciones financieras- envían a sus empleados a perfeccionarse en aquellas, aunque su participación en la generación de recursos humanos con un alto nivel de producción científico, casi nos atrevemos a decir que brilla por su ausencia; y b) coincidiendo con el Rector de la Universidad Nacional del Comahue, Dr. Jorge Rabassa, quien apuntó -a finales de mayo de 1999- que aunque fuera "fuerte" decirlo, "se trata de un negocio escandaloso, pero es obvio que el objetivo de las universidades privadas es exclusivamente el lucro, un lucro pleno, porque se gasta lo menos posible y se cobra lo más que se pueda». Más arriba hablamos de las corresponsalías que la Universidad Católica Argentina en el interior del país, el Rector Rabassa prefiere hablar de "franquicias", término quizá mucho más adecuado al lenguaje moderno del mercadeo. Cuando transcribimos sus dichos de que gastan poco dinero, debe tenerse presente que las estos institutos de educación superior no son más que eso, es decir, lugares de enseñanza -o enseñaderos, como alguna vez dijera el ex Presidente Alfonsín- ya que su oferta educativa apunta a las carreras tradicionales que tienen un mercado asegurado y a las cuales no atienden muchas de las universidades nacionales del interior del territorio, a la vez que ellas ignoran olímpicamente a las tareas de investigación, la extensión universitaria, los servicios y el montaje de costosos laboratorios, cosas éstas que definen per se al sentido y ser de las universidades (Rodríguez Kauth, 1997).

Al respecto, el propio Rector Rabassa abundó con otros comentarios como que «... mientras que a la universidad pública poner en marcha una nueva carrera académica le significa, además de un enorme esfuerzo académico y económico, esperar años -en ocasiones- para lograr la aprobación del Ministerio de Educación, estas privadas obtienen, hasta para la carrera de Medicina, habilitaciones provisorias por cinco años sin ningún tipo de control "; a todo lo cual agregó en otro párrafo que esto de la habilitación de múltiples sedes resulta irritante, ya que "... muchas veces reciben financiación del Estado nacional y de las provincias, mientras se niegan recursos a las universidades públicas".

A su vez las fundaciones, $\mathrm{u}$ otras formas de organización semejantes, convocaron a profesionales y científicos con formación académica definida como de alto nivel; los mismos debían estar capacitados en el exterior, especialmente en países de habla anglosajona, tener -obviamente- título de grado y, sobre todo, de posgraduados logrados en prestigiosas universidades extranjeras, tales como las de Harvard, Columbia, Oxford, Chicago, Cambridge, etc. Estos títulos eran -y son- requeridos fundamentalmente para las posiciones- en la carrera empresaria -de relieve en las áreas tales como la economía, la contabilidad y las diversas ramas de la ingeniería que apuntalan el desarrollo empresario. 
Lo relevante de estas organizaciones que funcionan como fundaciones, que con el transcurso del tiempo fueron creciendo en número e importancia, es que su influencia no se agota en la formación de recursos humanos para la satisfacción de las necesidades de la esfera que corresponde al mundo de la producción. También sus integrantes-por un efecto de transferencia-fueron los que paulatinamente comenzaron a introducirse en los ámbitos de los gobiernos nacional y provinciales y, de tal suerte que ellos son los que elaboraron -y elaboran- la mayor parte de los lineamientos políticos de los gobiernos nacionales que se fueron sucediendo desde entonces, ya hayan sido democráticos o dictatoriales, llegando a formar parte del capital humano que luego ocuparán lugares relevantes en los gabinetes ministeriales y en otras funciones de gobierno que con justicia deben ser consideradas estratégicas para el interés nacional.

Como muestra de lo dicho, basta recordar los nombres de los dos últimos ministros de economía del menemismo, Domingo F. Cavallo y Roque Fernández, cuyo lugar de origen -antes del lanzamiento a la esfera política- fueron los cursos de postgrado tomados en alguna de las Universidades mencionadas en Estados Unidos y, posteriormente, sus plataformas de lanzamiento fueron la Fundación Mediterráneo y la del CEMA, respectivamente, para sus meteóricas carreras políticas.

Lo que caracteriza a las organizaciones mencionadas es el notable apoyo financiero que ellas mismas reciben de los sectores de la producción, lo cual facilita el quehacer científico y tecnológico y posibilita a sus miembros recibir ingresos muy superiores a los de cualquier profesor universitario. Al efecto, recordar los dichos del ex súper Ministro de Economía, D. Cavallo, cuando afirmó que él no podía subsistir con una suma inferior a los 10.000 dólares mensuales, suma con la que colaboraba la fundación Mediterráneo a su modesto mantenimiento. Este es el mismo personaje que en su momento, ante un reclamo salarial de los trabajadores universitarios, los mandó textualmente a "lavar los platos», en un gesto de altanería y soberbia política de quien se sabía seguro del apoyo de su mentor sentado en la presidencia de la República.

Una de las diferencias entre estas fundaciones que actúan en el país, con instituciones similares de otros países, es la poca o escasa vinculación institucional que las mismas mantienen con universidades privadas o públicas, a pesar de que muchos de sus miembros se desempeñan simultáneamente como profesores en las mismas.

El desajuste entre los sectores de la producción y las universidades, a la vez que entre las organizaciones del tipo de las fundaciones a que hiciéramos referencia y las mismas universidades, no es un hecho casual desde la mirada de la ciencia política o sociológica. El empresariado argentino y los sectores de poder solo adjudican un valor productivo al conocimiento cuando el mismo -el conocimiento- se produce dentro de una determinada ideología y 
a los fines de satisfacer una demanda específica. En consecuencia, estos sectores de poder quieren tener sus propios centros y «obreros» de la producción de conocimientos. Centros y científicos que produzcan en la dirección del sentido que postula un determinado modelo social y económico y que operen totalmente aisladas de los ámbitos académicos, lugares donde todavía se tiene la pésima costumbre de manejar esquemas de pensamiento en los que se tiene en cuenta, entre otras cosas a la dignidad del hombre.

Por el contrario y como ya se dijo, los think tanks del capitalismo mundial trabajan en estrecha colaboración con las Universidades de élite y también con las empresas transnacionales y, lo que es más siniestro, si se compara con lo que ocurre en nuestro país, en estrecha colaboración con el Pentágono con el objetivo de colaborar con mano de obra barata para el desarrollo de técnicas sofisticadas para futuros emprendimientos bélicos que el dueño del Imperio pretenda llevar adelante.

\section{CONCLUSIONES}

Entendemos que las conclusiones generales se han ido desprendiendo del mismo relato que hemos venido exponiendo. Sin embargo, es preciso que puntualicemos un par de aspectos que nos han parecido notables:

a) Frente al empeño privatizador en que se manifiesta la cultura política contemporánea, los jóvenes -y los no tan jóvenes- hemos sabido salir a decir ¡basta!, aunque un poco tarde. La mentalidad egoísta e insolidaria solamente nos ha hecho movilizar de manera taxativa cuando un problema nos ha afectado en forma directa. Pero tampoco esto es semejante a nada, por el contrario, esto debería figurar como un estímulo para futuras acciones mancomunadas con otros sectores sociales.

b) La lucha de estos sectores universitarios no está aislada en nuestra América Latina. Junto con ella se han expresado los jóvenes estudiantes universitarios mexicanos, los cuales se vienen oponiendo de manera sistemática a la privatización de la enseñanza en su país y a los proyectos oligárquicos de la conducción nacional y académica de cobrar arancel a aquellos jóvenes. Alonso (1999) llega a una conclusión semejante a la nuestra cuando dice: «El vigoroso movimiento estudiantil de la primavera de 1999 es una muestra, ... Es un signo más de la resistencia a un modelo económico excluyente y otro grito de protesta ante el neoliberalismo y en contra del régimen de partido de Estado». No estamos solos en el mundo.

c) El episodio produjo que no solamente se reclamara ante los poderes políticos de la Nación, sino que también amplios sectores de la población estudiantil hicieran oír sus quejas al Rector Shuberoff de como 
está gastando los dineros públicos. Vale decir, la protesta no ha estado dirigida por intereses sectoriales o estrechos del partidismo político, apuntó mucho más alto.

Por último, si se tiene en cuenta que la Universidad Argentina, pese a los embates gubernamentales contra ella, debido a que en sus claustros se piensa, todavía es un espacio de resistencia para estas teorías y todo lo que ellas implican, es posible atreverse a pensar que los que vivimos en y de los espacios universitarios tenemos suerte, lo que aún no sabemos es cuanto ésta nos va a llegar a durar.

\section{BIBLIOGRAFÍA}

ALONSO, J.: «La lucha de los universitarios irrumpe en el escenario". Revista Envío, (Managua), Nro. 206, 1999.

FRIGERIO, G. y otros: Politicas, Instituciones y actores en educación. Ediciones Novedades Educativas, Buenos Aires, 1997.

GENTILI, P.: «¿La maldición divina? Las complejas relaciones entre los hombres de negocios y las políticas educativas». En Frigerio (1997).

INGENIEROS, J.: (1913) El Hombre Mediocre. Ediciones Mar Océano, Bs. Aires, Vol. 7, 1962

RODRIGUEZ KAUTH, A:: Lecturas psicopoliticas de la realidad nacional desde la izquierda. Centro Editor de América Latina, Bs. Aires, 1994.

RODRIGUEZ KAUTH, A.: «Lectura psicosocial del aula universitaria: ayer y hoy». Revista Universidades (México), Nro. 14, 1997.

RODRIGUEZ KAUTH, A.: Aguafuertes de fin de siglo. Editorial Almagesto, Bs. Aires, 1998.

RODRIGUEZ KAUTH, A.: «Del Estado empresario al Estado de las empresas". Revista Idea de la Facultad de Ciencias Humanas, San Luis, Nro. 26, $1998 b$.

\section{RESUMEN}

Los autores pasan revista a la relación entre la Universidad Argentina, durante la centuria que termina, con el poder político. La misma normalmente no transitó carriles armónicos, los mismos fueron particularmente encontrados en periodos de dictaduras militares y durante los gobiernos de signo justicialista.

Palabras clave: Universidad, política, Argentina. 


\section{ABSTRACT}

The authors examine the relationship between Argentina's state universities, during the twentieth century, and political power. This relationship was not always harmonious, and it was particularly conflictive during periods of military rule and also when the "Partido Justicialista» was in power.

Key words: University, politics, Argentina. 